\title{
Influence of intranasal steroids during the grass pollen season on bronchial responsiveness in children and young adults with asthma and hay fever
}

B J Thio, G L M Slingerland, A M Fredriks, A F Nagelkerke, R A Scheeren, H J Neijens, J J Roord, J E Dankert-Roelse

\begin{abstract}
Background-It has been reported that intranasal corticosteroids can influence bronchial hyperresponsiveness (BHR) in asthmatic subjects with seasonal rhinitis. The purpose of the present study was to evaluate the effect of intranasal fluticasone propionate and beclomethasone dipropionate on BHR and bronchial calibre (forced expiratory volume in one second, $\mathrm{FEV}_{1}$ ) in children and young adults with seasonal rhinitis and mild asthma during two consecutive grass pollen seasons.

Methods-In the first pollen season 25 patients aged 8-28 years were included in a double blind, placebo controlled study. The active treatment group used fluticasone aqueous spray $200 \mu \mathrm{g}$ once daily. In the second pollen season 72 patients aged 8-28 years participated in a double blind, placebo controlled study of a similar design to that of the previous year except that an additional treatment group of patients using beclomethasone $200 \mu \mathrm{g}$ twice daily was included. FEV $_{1}$ was measured before and after three and six weeks of treatment; BHR to methacholine $\left(\mathbf{P D}_{20}\right)$ was measured before and after six weeks of treatment.
\end{abstract}

Results-In the first season the mean (SD) $\log \mathrm{PD}_{20}$ of the patients decreased significantly both in the fluticasone group (from $2.43(0.8) \mu \mathrm{g}$ to $1.86(0.85) \mu \mathrm{g})$ and in the placebo group (from $2.41(0.42) \mu$ g to 1.87 $(0.78) \mu \mathrm{g})$ without any intergroup difference in the change in $\log P D_{20}$. In the second pollen season the mean $\log P D_{20}$ in the fluticasone, beclomethasone, and placebo groups did not change significantly.

Conclusions-Intranasal steroids did not influence BHR during two grass pollen seasons in children and young adults with seasonal rhinitis and mild asthma.

(Thorax 2000;55:826-832)

Keywords: bronchial hyperresponsiveness; corticosteroids; allergic rhinitis; asthma

A beneficial influence of treatment with intranasal corticosteroids on bronchial hyperresponsiveness (BHR) in patients with seasonal allergic rhinitis has been observed, ${ }^{12}$ although other studies have been unable to confirm this finding. ${ }^{3}$ It has been suggested that the beneficial influence of intranasal steroids on the lower airways is due to a restoration of the nasal function leading to an improvement in air conditioning (filtering, warming, and humidifying the air). ${ }^{5}$ Other putative mechanisms are a reduction of aspiration of postnasal secretions, an alleviation of upper/lower airway reflexes, and a reduction of reabsorption of mediators or chemotactic factors from the inflammatory process in the nose or sinuses.

Fluticasone propionate (FP) and beclomethasone dipropionate (BDP) are effective steroids in the treatment of seasonal allergic rhinitis. ${ }^{6-10}$ FP possesses twice the antiinflammatory potency of BDP, as measured by vasoconstrictor assay, ${ }^{6}$ and is effective as a once daily dosage regimen ${ }^{10}$ in half the dose of BDP. ${ }^{6}$ The pharmacokinetic properties of FP and BDP are different. The oral bioavailability of intranasal FP is lower than BDP because of extensive first pass metabolism and negligible gastrointestinal absorption. ${ }^{11}$ This is important for an intranasally administered drug as a substantial part of it $(48-78 \%)$ is swallowed. ${ }^{12}$

In two consecutive years we studied whether the use of intranasal FP or BDP during the grass pollen season could influence BHR, bronchial calibre, and airway symptoms in children and young adults with mild asthma and seasonal allergic rhinitis.

\section{Methods}

STUDY DESIGN

Both studies were placebo controlled, randomised, and double blind. Treatment was started in the month of May when pollen counts were expected to start rising within two weeks (figs 1 and 2). A forecast on the beginning of the pollen season was given from the University Hospital in Leiden. In the Netherlands the grass pollen season may start anywhere between the beginning of May and the first half of June and may last for up to three months.

STUDY AND RESCUE MEDICATION

In the first season (1994) patients received either $200 \mu \mathrm{g}$ FP aqueous spray or placebo aqueous spray. Patients were carefully instructed to use two actuations of FP or placebo aqueous spray containing $50 \mu \mathrm{g}$ per actuation in each nostril once daily for six weeks. Patients used the nasal spray in the morning after awakening. 
In the second season (1995) there were three study groups. Patients received $200 \mu \mathrm{g} \mathrm{FP}$ aqueous spray, $400 \mu \mathrm{g}$ BDP aqueous spray, or placebo aqueous spray. Patients in the FP group received $\mathrm{FP}$ aqueous morning spray and placebo evening spray. Patients in the BDP group received BDP aqueous spray as both morning and evening spray. Patients in the placebo group received two bottles of placebo aqueous spray. The FP and BDP aqueous sprays both contained $50 \mu \mathrm{g}$ per actuation. All patients were instructed to use two actuations in each nostril twice daily for a period of six weeks. The treatments were dispensed by the manufacturer and were not distinguishable from each other. Patients were instructed to use the nasal spray in the morning after awakening and in the evening before bedtime. They were instructed to inspire to total lung capacity before spraying the medication as a safeguard against aerosol delivery into the lower airways. Concurrent medication (including intranasal vasoconstrictors and oral and/or topical anti-

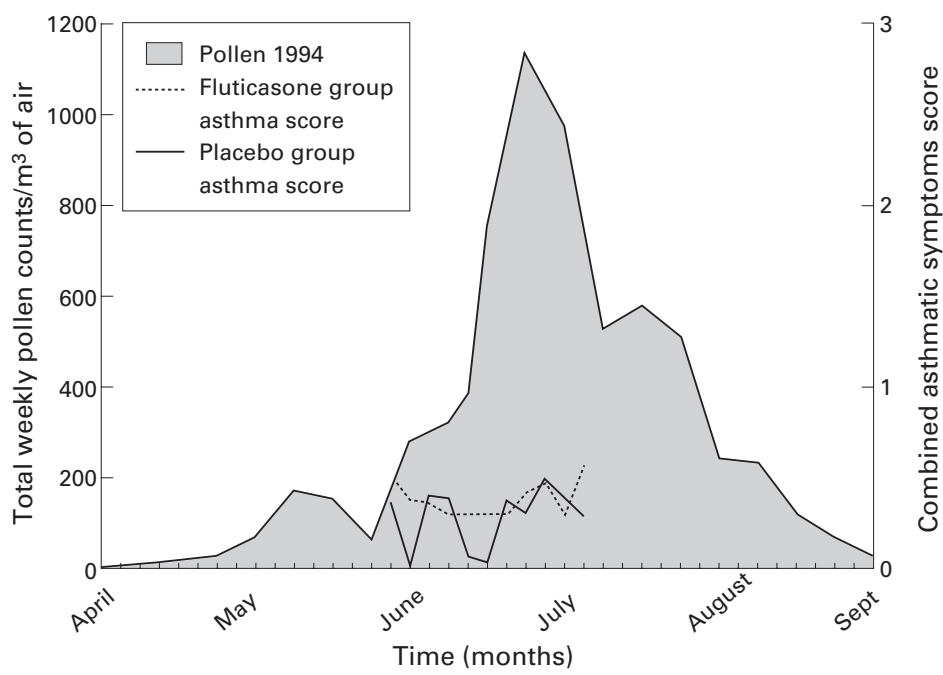

Figure 1 Combined asthma symptom scores in relation to pollen counts during treatment with intranasal fluticasone (FP) or placebo in the grass pollen season of 1994. Asthma symptoms were not significantly different between groups.

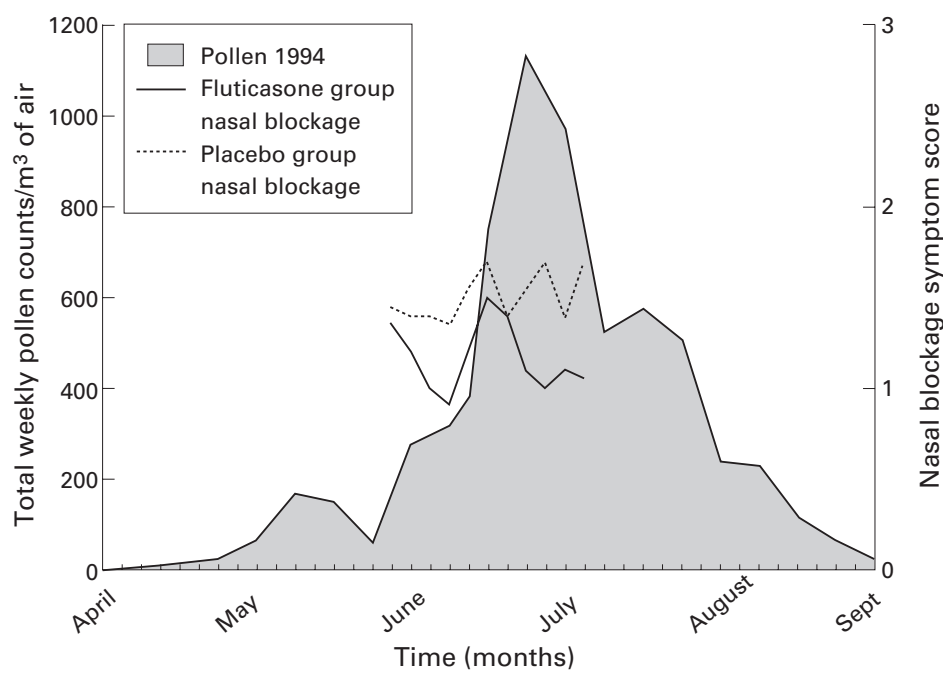

Figure 2 Nasal blockage symptom scores in relation to pollen counts during treatment with intranasal fluticasone (FP) or placebo in the grass pollen season of 1994. Nasal blockage was not significantly different between groups. histamines) was not allowed during the study except for salbutamol $200 \mu \mathrm{g}$ Rotadisks for asthmatic symptoms and levocabastine eye drops for symptoms of allergic conjunctivitis. Both salbutamol and levocabastine were taken as needed.

SUBJECTS

In the first season 25 patients and in the second season 72 patients aged 8-28 years were included in the study. All patients had a clinical history of hay fever and mild asthma according to ATS criteria ${ }^{13}$ with worsening of asthma symptoms in the summer months. All patients had a positive IgE RAST ( $>0.7 \mathrm{kU} / 1$; Pharmacia CAP System RAST FEIA) for grass pollen or a positive skin prick test (wheal diameter $>0.4$ wheal diameter of the histamine control) on an aqueous grass pollen extract of $10000 \mathrm{BU} / \mathrm{ml}$ (Vivodiagnost ALK, Benelux). Birch pollen and house dust mite allergies were also assessed. Patients with allergy to birch pollen and/or house dust mite were not excluded from the study. None of the patients had used inhaled, intranasal, and/or oral steroids in the three months before the study. Patients using theophyllines, anticholinergic agents, long acting bronchodilators, and cromoglycate for pulmonary or nasal use and those receiving allergy immunotherapy were excluded. Each subject was in a clinical steady state and had not reported symptoms of upper or lower respiratory tract infection for at least three weeks before the study. Those with nasal and/or lung disease different from allergic rhinitis and asthma were excluded.

The medical ethics committee of the University Hospital Vrije Universiteit approved the study protocol. Patients and/or parents gave informed consent to participate in the study.

DAILY DIARIES

Symptom scores were recorded in the morning to evaluate night time symptoms and in the evening to evaluate symptoms during the day. Recorded symptoms were shortness of breath, wheezing, cough and nasal blockage, and each was scored as $0=$ none, $1=$ mild, $2=$ moderate, and $3=$ severe. In the second season patients also recorded sneezing and runny noses. All patients recorded use of salbutamol which the investigator checked by counting the used blister packs at each hospital visit.

\section{METHACHOLINE CHALLENGE}

Patients arrived in the hospital having abstained from inhaled bronchodilators for at least eight hours. Methacholine challenge was performed to measure BHR according to the guidelines of Birnie et al. ${ }^{14}$ Before every challenge test three reproducible baseline measurements of forced expiratory volume in one second $\left(\mathrm{FEV}_{1}\right)$ and forced vital capacity (FVC) were obtained with a SensorMedics Pulmonet III computerised water spirometer (IBM PS 235X). The best $\mathrm{FEV}_{1}$ and FVC values were taken and Zapletal reference values were used to calculate the percentage predicted value of $\mathrm{FEV}_{1}$. Aerosol dispersed methacholine bromide in unbuffered saline was given in 
doubling concentrations $(0.15-160 \mathrm{mg} / \mathrm{ml})$. The aerosol was generated by a De Vilbiss 646 nebuliser (De Vilbiss Co, Somerset, PA, USA) which was operated with $3 \mathrm{ml}$ solution in the nebuliser cup. The nebuliser was attached to a Rosenthal-French dosimeter (Laboratory for Applied Immunology, Fairfax, VA, USA) driven by air at $137.8 \mathrm{kPa}(20 \mathrm{psi})$ with a time adjustment of 0.6 seconds. Aerosol delivery was performed according to Verberne et al. ${ }^{15}$ To exclude reactions to the diluent, saline solution was inhaled before methacholine in a similar manner. $\mathrm{FEV}_{1}$ was measured in triplicate three minutes after saline solution or methacholine inhalation. The interval between consecutive doses was five minutes. The next methacholine dose was not given if $\mathrm{FEV}_{1}$ had fallen below $80 \%$ of baseline. $\mathrm{PD}_{20}$ was calculated from a log dose-response plot with linear interpolation of data points.

\section{POLLEN COUNT}

The study was performed during the grass pollen seasons of 1994 and 1995. Airborne pollen grains were sampled daily by a Burkard volumetric pollen trap (Rickmansworth, UK) at the University Hospital in Leiden. The distance between Amsterdam and Leiden is 25 miles. Grass pollen counts were expressed as the total weekly count of grains per cubic meter of air.

\section{COMPLIANCE}

In the second season compliance with treatment was checked. Twenty morning and evening bottles containing study medication were weighed with a Mettler PC 4400 Delta Range weighing machine before being handed out to patients. The mean weight delivered per actuation according to the analysis of the manufacturer was $98.2 \mathrm{mg}$ (FP), $103 \mathrm{mg}$ (BDP), and $98.2 \mathrm{mg}$ (placebo). We calculated the mean used actuations per day. All patients were instructed to use eight actuations per day. Patients taking more than $70 \%$ (5.6 actuations) or less than $130 \%$ (10.4 actuations) of the total prescribed amount of treatment were considered compliant. ${ }^{16}$

\section{STUDY SCHEME}

The study started with a screening visit in which clinical history was obtained and physical examination performed. At the screening visit patients were provided with a diary for recording asthma and nasal symptoms. Use and inhalation technique of salbutamol was explained and/or checked. At the second visit (within two weeks of the first) a methacholine challenge test was performed to measure BHR. When all inclusion criteria were met, patients were randomised into a study group. Patients started the treatment period simultaneously, when pollen counts were expected to start rising within two weeks. During the third visit (three weeks after the start of the treatment period) $\mathrm{FEV}_{1}$ was measured and patients received a new supply of study medication. A second methacholine challenge test was performed at visit 4 (six weeks after the start of the treatment period). In the second study the study medication and rescue medi- cation were returned to check for compliance at visits 3 and 4 .

ANALYSIS OF DATA

All methacholine $\mathrm{PD}_{20}$ values were logarithmically transformed before analysis. In the first season comparison of subject characteristics at baseline between groups was analysed using the Student's test for independent groups with the exception of the analysis of proportion of allergy between groups which was performed with a $\chi^{2}$ test. The changes in $\mathrm{FEV}_{1} \%$ predicted and $\mathrm{PD}_{20}$ within treatment allocation groups were analysed using a Student's $t$ test for paired measurements. Between group changes were analysed with a Student's $t$ test for independent groups. The difference in the proportion of each group that changed more than one doubling dose was analysed using Fisher's exact test. Mean morning and evening symptom scores for nasal blockage and asthma were calculated for a period of 16 days in the first three weeks of the treatment period and a period of 16 days in the second three weeks of the treatment period. We took the middle 16 days instead of 21 days of each treatment period because most of the patients filled out the first and last days of the diary incompletely. Symptom scores and the use of salbutamol were analysed with MANOVA for repeated measurements. If a score was missing an interpolation was performed to calculate a score.

In the second season ANOVA was used to evaluate baseline characteristics between treatment groups, with the exception of the analysis of proportion of allergy between groups which was performed by $\chi^{2}$ testing. The changes in $\mathrm{FEV}_{1} \%$ predicted and $\mathrm{PD}_{20}$ within treatment allocation groups were analysed with the Student's $t$ test for paired measurements. ANCOVA was used to evaluate treatment efficacy on $\mathrm{PD}_{20}$ against placebo with baseline $\mathrm{PD}_{20}$ and age as covariant. Age was taken as covariant because the mean age in the BDP group was significantly lower than in the other groups. ANOVA was used to evaluate treatment efficacy on $\mathrm{FEV}_{1}$ against placebo. The symptom scores were analysed with MANOVA for repeated measurements. If a score was missing an interpolation was performed to calculate a score. To evaluate compliance the differences between the estimated number of actuations used and the number of actuations prescribed were analysed with the one sample $t$ test. The difference in compliance between the treatment groups was analysed with a Student's $t$ test for independent groups. In both seasons a $\mathrm{p}$ value of $<0.05$ was considered statistically significant.

\section{Results}

In the first season four of the 25 patients dropped out of the study, two from each study group. One patient dropped out of the placebo group because of uncontrollable symptoms and three patients because of non-compliance with the protocol. Twenty one patients were therefore included in the final analysis (table 1). Eleven patients were randomised to the FP group. There were significantly more patients 
Table 1 Mean (SD) characteristics of study subjects

\begin{tabular}{|c|c|c|c|c|c|c|c|}
\hline & \multicolumn{3}{|l|}{1994} & \multicolumn{4}{|l|}{1995} \\
\hline & $\begin{array}{l}F P \\
(n=11)\end{array}$ & $\begin{array}{c}\text { Placebo } \\
(n=10)\end{array}$ & $\begin{array}{l}p \\
\text { value }^{*}\end{array}$ & $\begin{array}{l}F P \\
(n=25)\end{array}$ & $\begin{array}{l}B D P \\
(n=23)\end{array}$ & $\begin{array}{r}\text { Placebo } \\
(n=24)\end{array}$ & $\begin{array}{l}p \\
\text { value }\end{array}$ \\
\hline Age (years) & 18.4 & 16.2 & NS & 20.5 & 17.1 & 19.7 & 0.03 \\
\hline $\mathrm{F}: \mathrm{M}$ & $7: 4$ & $6: 4$ & NS & $16: 9$ & $14: 9$ & $15: 9$ & NS \\
\hline $\mathrm{FEV}_{1}(\%$ pred) & $109(10)$ & $102(15)$ & NS & $104(17)$ & $101(16)$ & $100(10)$ & NS \\
\hline $\log \mathrm{PD}_{20}(\mu \mathrm{g})$ & $2.43(0.8)$ & $2.41(0.4)$ & NS & $2.24(0.9)$ & $2.02(0.9)$ & $2.29(0.8)$ & NS \\
\hline Birch pollen ${ }^{\star}$ & $91 \%$ & $70 \%$ & NS & $64 \%$ & $57 \%$ & $42 \%$ & NS \\
\hline
\end{tabular}

$\mathrm{FP}=$ fluticasone propionate $\mathrm{BDP}=$ beclomethasone dipropionate $\mathrm{FEV}_{1}=$ forced expiratory volume in one second; $\mathrm{PD}_{20}=$ dose of methacholine causing a fall in $\mathrm{FEV}_{1}$ of $20 \%$ or more; $\mathrm{NS}=$ not significant.

${ }^{\star} \mathrm{p}$ values from 1994 are based on the Student's $t$ test for independent groups and from 1995 on ANOVA, except those for birch pollen which are based on $\chi^{2}$ testing for both years.

Table 2 Mean (SD) changes in FEV $\%$ predicted

\begin{tabular}{lrlll}
\hline & Pretreatment & Mid treatment & Post treatment & pvalue * $^{*}$ \\
\hline Fluticasone & $103.6(16.9)$ & $111.7(6.0)$ & $102.8(8.0)$ & $<0.001$ \\
Beclomethasone & $100.7(16.2)$ & $107.7(5.1)$ & $103.1(5.5)$ & $<0.01$ \\
Placebo & $99.9(10.1)$ & $102.8(4.7)$ & $100.4(5.3)$ & $\mathrm{NS}$ \\
\hline
\end{tabular}

^Difference in pretreatment and mid treatment values.

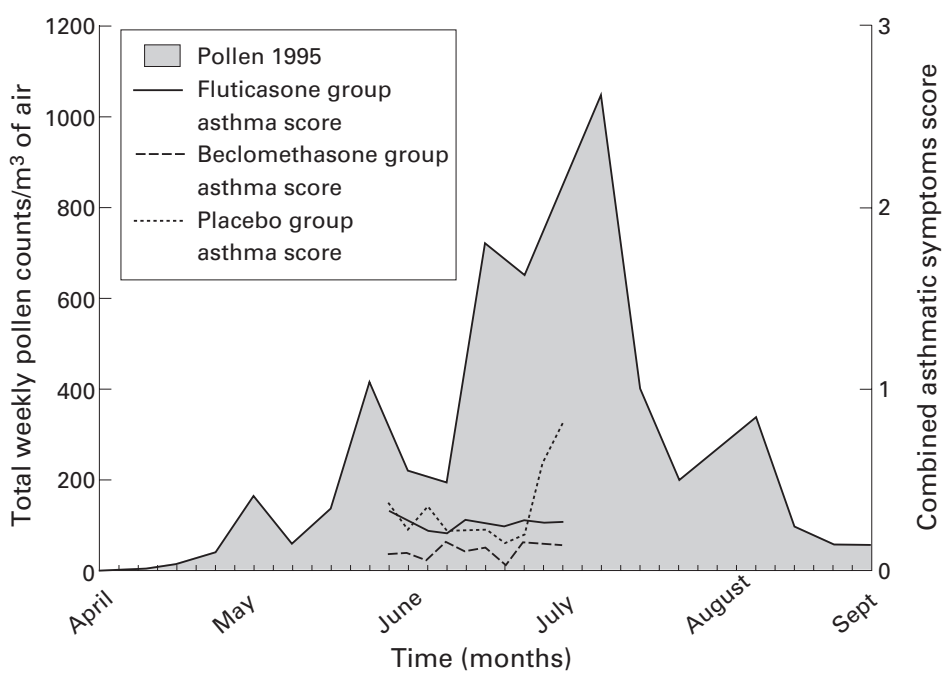

Figure 3 Combined asthma symptom scores in relation to pollen counts during treatment with intranasal fluticasone (FP), beclomethasone (BDP), or placebo in the grass pollen season of 1995. Asthma symptoms were not significantly different between groups.

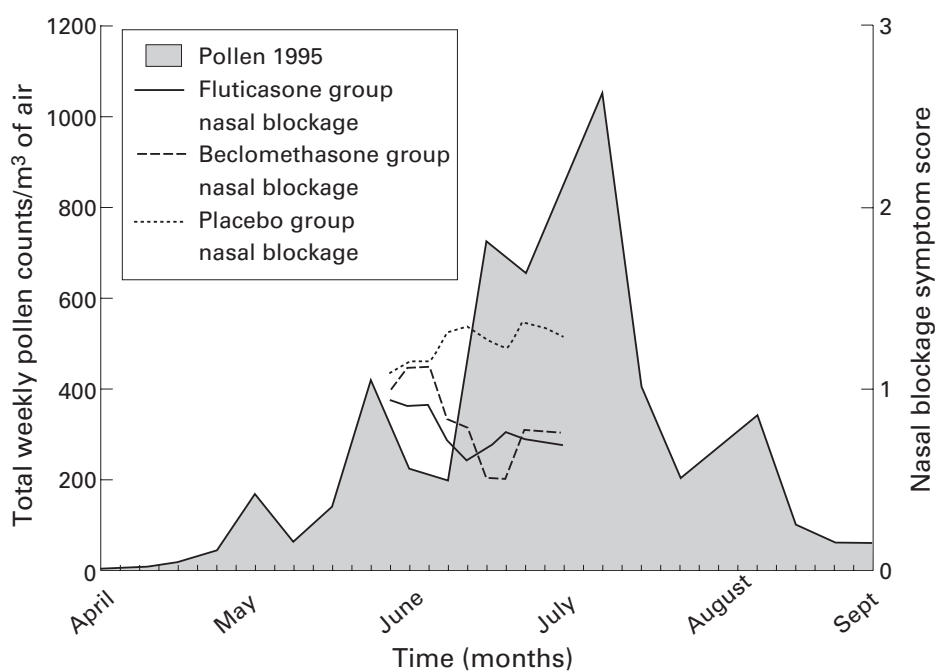

Figure 4 Nasal blockage symptom scores in relation to pollen counts during treatment with intranasal fluticasone (FP), beclomethasone (BDP), or placebo in the grass pollen season of 1995. Nasal blockage was significantly less in the FP and BDP groups than in the placebo group $(p=0.001)$. in the FP group with allergy to house dust mite. There were no significant differences in any of the other characteristics.

In the second season five of the 72 patients dropped out, two from the BDP group and three from the placebo group, all because of non-compliance with the protocol. No patients dropped out from the FP group. Sixty seven patients were therefore included in the final analysis. Twenty five patients were randomised to the FP group, 23 to the BDP group, and 24 to the placebo group. The characteristics of the subjects at randomisation were not different, except that the mean age of patients in the BDP group was on average 3.4 and 2.6 years lower than those in the FP and placebo groups, respectively $(\mathrm{p}=0.03$, table 2$)$.

\section{POLLEN COUNTS}

Mean weekly pollen counts in the pollen seasons of 1994 and 1995 are shown respectively in figs 1 and 3 . In the first season grass pollen counts increased during the treatment period. Measurements after the treatment period were, for most patients, just after the peak of the pollen season. In the second season grass pollen counts in the initial three weeks were at a relatively low level. Thereafter, pollen counts increased quite steeply until approximately $1000 / \mathrm{m}^{3}$. Final measurements started at the peak of the pollen season and lasted for two weeks.

\section{SYMPTOM SCORES}

The scores for nasal blockage and for combined asthma symptoms (wheezing, shortness of breath, and cough) in relation to pollen counts of both seasons are shown in figs $1,2,3$, and 4 .

In the first season mean daily asthma scores were less than mild (score 1): the wheezing score was less than 0.5 , shortness of breath ranged between 0.5 and 0.8 , and cough scores ranged between 0.5 and 0.8 . Mean scores for wheezing, shortness of breath, cough, and use of salbutamol did not vary significantly between the groups (fig 1). Nasal blockage ranged between mild and moderate (score 1 and 2) and tended to be lower in the FP group, although there was no significant difference between the groups during the treatment period (fig 2).

In the second season mean evening scores for wheezing were low (mean score in all three groups less than 0.3 ) and significantly less in the FP and BDP groups than in the placebo ( $p$ $=0.024)$. The mean daytime scores for cough, shortness of breath, and use of salbutamol did not differ significantly between the groups (fig 3). Nasal blockage, sneezing, and runny noses in the evening were significantly less in the FP and BDP groups than in the placebo group ( $\mathrm{p}$ $=0.001, \mathrm{p}=0.005$, and $\mathrm{p}=0.038$, respectively, fig 4).

BRONCHIAL HYPERRESPONSIVENESS

In the first season the pre-trial level of the $\log \mathrm{PD}_{20}$ did not differ significantly between groups $(p=0.98)$. The mean $(S D) \log \mathrm{PD}_{20}$ of the patients decreased significantly both in the 

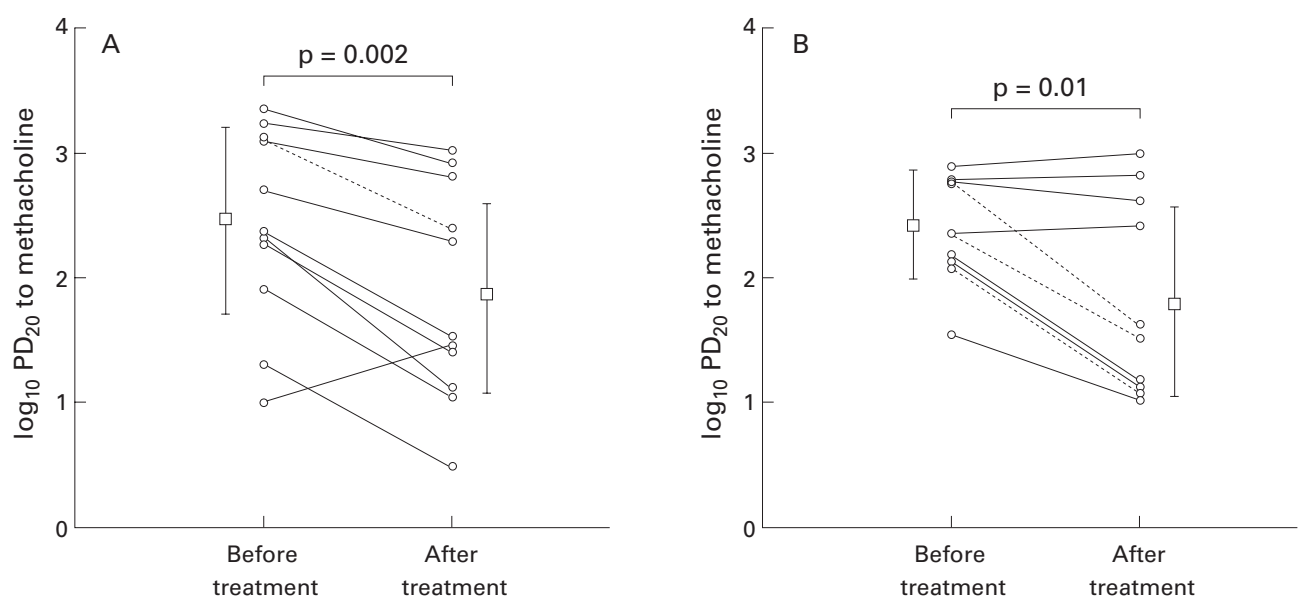

Figure 5 Change in individual log $P D_{20}$ values during the grass pollen season of 1994 in $(A)$ patients treated with FP and (B) the placebo group. Broken lines = patients without birch pollen allergy.

FP group (from $2.43(0.8) \mu \mathrm{g}$ to 1.86 $(0.85) \mu \mathrm{g}, \mathrm{p}=0.002)$ and in the placebo group (from $2.41(0.42) \mu \mathrm{g}$ to $1.87(0.78) \mu \mathrm{g}, \mathrm{p}=$ 0.01 ) without any intergroup difference in the change in $\log \mathrm{PD}_{20}\left(\mathrm{p}=0.2\right.$, fig 5). The $\log \mathrm{PD}_{20}$ decreased by more than one doubling dose in all but one patient in the FP group and in six of 10 patients in the placebo group. This difference between the groups was not significant $(\mathrm{p}=0.38)$.

The only patient in the FP group without allergy to birch pollen had a decrease in $\mathrm{PD}_{20}$ of more than one doubling dose. All four patients in the placebo group who did not have a decrease of $\mathrm{PD}_{20}$ were allergic to birch pollen.

In the second season pretreatment levels of $\log \mathrm{PD}_{20}$ did not differ significantly between the study groups $(\mathrm{p}=0.52)$. The $\log \mathrm{PD}_{20}$ tended to increase during the treatment period in all three study groups (fig 6), but the changes were not significantly different within or between groups $(p=0.97)$. The changes in mean $(S D)$ $\log \mathrm{PD}_{20}$ in the FP, BDP, and placebo group were, respectively, $2.24(0.9) \mu \mathrm{g}$ to 2.39 $(0.8) \mu \mathrm{g}, 2.02(0.9) \mu \mathrm{g}$ to $2.23(0.8) \mu \mathrm{g}$, and $2.29(0.8) \mu \mathrm{g}$ to $2.54(0.9) \mu \mathrm{g}$. When patients with an allergy to grass pollen only were analysed the changes in $\log \mathrm{PD}_{20}$ between the groups were also not significantly different.

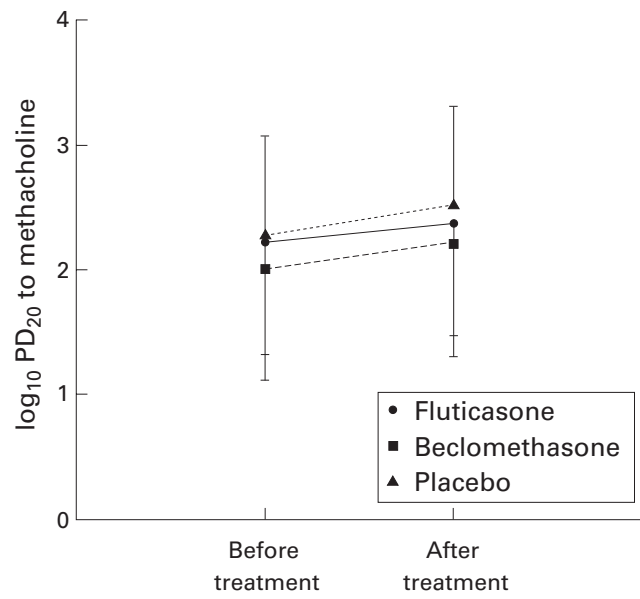

Figure 6 Change in mean log $P D_{20}$ values during the grass pollen season of 1995 .
BRONCHIAL CALIBRE

In the first season the mean \% predicted $\mathrm{FEV}_{1}$ at baseline was not significantly different in the FP and placebo groups (table 1) and did not change during or after treatment in the FP (baseline 109 (10)\%, mid treatment 110 (9.1)\%, end of treatment $114(10) \%$ ) or placebo groups (baseline 102 (15)\%, mid treatment 101 (16)\%, end of treatment 102 (16)\%).

In the second season the mean $\%$ predicted $\mathrm{FEV}_{1}$ at baseline was not significantly different in the three groups (table 2) but increased in all three groups during treatment, which was significant in the FP group $(p<0.001)$ and the BDP group $(p<0.01)$ but not in the placebo group $(p=0.1)$. There was no significant difference in the change in $\%$ predicted $\mathrm{FEV}_{1}$ between the groups during or at the end of treatment (table 2).

COMPLIANCE

All of the patients in the FP group returned their medication but three patients in the BDP group and four in the placebo group did not. The percentage of compliant patients (taking more than $70 \%$ or less than $130 \%$ of the total prescribed amount of treatment) was $62.3 \%$; the percentage of non-compliant patients was $35.8 \%$. One patient used more than $130 \%$ of the prescribed medication. There was no significant difference in compliance between the three treatment groups $(\mathrm{p}>0.1)$.

\section{Discussion}

We investigated whether the use of an intranasal corticosteroid can modify BHR during the grass pollen season in patients with mild asthma and seasonal allergic rhinitis. The effect of FP on BHR was studied in one season and the effect of equipotent doses of FP and BDP was studied in the next season. In the first season we found a significant and equal increase in BHR in patients in both the FP and placebo groups. In the next season we observed no increase in BHR in patients given placebo nor in those treated with BDP or FP. As expected, we found a decrease in nasal symptoms with intranasal corticosteroids. Rather unexpectedly, 
we found an improvement in \% predicted $\mathrm{FEV}_{1}$ under low allergen exposure in the patients treated with BDP or FP. Thus, intranasal steroids were not able to prevent the increase in BHR which occurred in the first season nor to reduce $B H R$ in the second season.

The increase in BHR during the grass pollen season is thought to be caused by a gradual cumulative effect of deposition of small amounts of allergen in the lower airways. It has been suggested that a certain threshold of pollination is required to increase $\mathrm{BHR},{ }^{17}$ although the relationship between allergen dose and effect on BHR has not been sufficiently well elucidated. In the first season we observed an overall increase in BHR of almost two doubling doses. A similar increase in BHR during the pollen season has been observed in asthmatic subjects with seasonal allergic rhinitis, ${ }^{18} 19$ although this was not found in all studies. ${ }^{2021}$ In the second season we observed no increase in BHR.

We assume that the pollination threshold leading to change in BHR was reached in the treatment period during the first season. During the second season the pollination may not have progressed sufficiently in the treatment period, hence the absence of a detectable increase in BHR in any of the three groups.

Four studies have assessed the effect of intranasal steroids on BHR during the pollen season. ${ }^{1-4}$ In one of these studies all patients had asthma ${ }^{2}$ and this study found a significant effect of intranasal steroid on BHR. Two studies selected patients without clinically evident asthma, ${ }^{13}$ one of which found a significant effect of intranasal steroid on $\mathrm{BHR}^{1}$ while the other one did not. ${ }^{3}$ Armitage et $a l^{4}$ included patients with and without asthma and did not find an effect of intranasal steroid on BHR.

An important difference between our study and the others assessing the effect of intranasal steroids on BHR during the pollen season ${ }^{1-4}$ is the use of nasal rescue medication such as antihistamines which we did not allow to avoid interference with the effects of the intranasal steroids. Although antihistamine monotherapy does not influence BHR to methacholine, ${ }^{22}{ }^{23}$ and only has a small bronchodilator effect, ${ }^{24}$ a reduction in both nasal and asthma symptoms was recently reported in two large multicentre trials of the effects of antihistamines on seasonal asthma. ${ }^{25}{ }^{26}$ Additional treatment with antihistamines may influence the lower airways either directly or by an improvement in nasal function, and may account for the inconsistent observations of the effects of intranasal steroids on BHR in the literature.

There are at least three other explanations for the inconsistent observations. The impact of the treatment of allergic rhinitis with intranasal steroids on BHR may depend on the pollen load. A significant effect of intranasal steroids on the lower airways may only be detectable under certain pollen conditions. High pollen concentrations may overwhelm the effect of intranasal steroids on the lower airways, and low pollen concentrations may mask the difference between treatment and control groups.
Secondly, the patient group studied may play a role. It may be possible to influence BHR in patients with allergic rhinitis and mild asthma, but not in patients with BHR without clinically evident asthma or in patients with more than mild asthma. Furthermore, it may be that BHR can only be influenced in patients with severe allergic rhinitis.

Thirdly, we cannot exclude the possibility that the use of intranasal steroids in higher doses or for longer than six weeks can affect BHR.

Patients with birch pollen allergy in addition to grass pollen allergy were not excluded from the study, although this could have introduced a confounding effect. However, we found that patients with birch pollen allergy showed the same pattern of change in BHR as those without allergy to birch pollen.

We observed an increase in airway calibre in the three treatment groups halfway through the treatment period in the second pollen season, which was significant in the FP and the BDP groups. However, the increase in airway calibre in those receiving steroids was not significantly different from the increase in the placebo group. The effect of intranasal steroids on airway calibre is unlikely to be clinically significant as the trend in change in airway calibre was only evident during treatment and not after the treatment period. Moreover, this effect has not been observed in previous studies, although smaller groups were used than in our study.

As expected, nasal symptom scores were lower in patients treated with intranasal steroids than in those receiving placebo, although this only reached significance in the second season when larger study groups were used. Asthmatic symptom scores were less than mild in all patients in both seasons. A trend towards higher asthmatic symptom scores was observed towards the end of the treatment period in the second season in the placebo group compared with the groups treated with intranasal steroids. In one previous study intranasal steroids reduced asthma symptoms ${ }^{5}$ during the pollen season in asthmatic subjects with allergic rhinitis but Corren et at reported no such effect.

Compliance between groups was not different, suggesting that this factor did not influence the results. In the second season we found that almost two thirds of the patients consumed more than $70 \%$ of the prescribed amount of treatment, which is a comparable rate of compliance to that in earlier studies with nasal spray medication. ${ }^{27}$

In conclusion, pollen allergen load appears to be related to changes in BHR. Despite a reduction in nasal symptoms, the use of intranasal steroids did not influence BHR during two consecutive grass pollen seasons in young patients with seasonal allergic rhinitis and mild asthma.

The authors thank Dr F Th M Spieksma for the grass pollen count and Nilofer Sabrine for her help. Financial support for the study was provided by GlaxoWellcome Netherlands. 
1 Foresi A, Pelucchi, Gherson G, et al. Once daily intranasal fluticasone propionate $(200 \mu \mathrm{g})$ reduces nasal symptoms
and inflammation but also attenuates the increase in bronand inflammation but also attenuates the increase in bronchial responsiveness during the pollen season
rhinitis. F Allergy Clin Immunol 1996;98:274-82.

2 Corren J, Adinof AD, Buchmeier AD, et al. Nasal beclomethasone prevents the seasonal increase in bronchial responsiveness in patients with allergic rhinitis and asthma. f Allergy Clin Immunol 1992;90:250-6.

3 Pelucchi A, Chiapparino, Mastropasqua B, et al. Effects of intranasal azelastine and beclomethasone dipropionate on nasal symptoms, nasal cytology, and bronchial responsiveness to methacholine in allergic rhinitis in response to grass pollens. F Allergy Clin Immunol 1995;95:515-23.

4 Armitage JM, Sin Fai Lam K, Wilkinson, et al. Investigation of the tendency to wheeze in pollen sensitive patients. Clin Exp Allergy 1992;22:916-22.

5 Welsh PW, Stricker WE, Chu-Pin Chu, et al. Efficacy of beclomethasone nasal solution, flunisolide, and cromolyn in relieving symptoms of ragweed allergy. Mayo Clin Proc 1987;62:125-34.

6 Ratner PH, Paull BR, Findlay SR, et al. Fluticasone propionate given once daily is as effective for seasonal allergic rhinitis as beclomethasone dipropionate given twice daily. F Am Cancer Inst 19992;90:285-91.

7 Nathan RA, Bronsky EA, Fireman P, et al. Once daily fluticasone aqueous nasal spray is an effective treatment for seasonal allergic rhinitis. Ann Allergy 1991;67:332-8.

8 Meltzer EO, Orgel HA, Bronsky EA, et al. A dose ranging study of fluticasone propionate aqueous nasal spray fo seasonal allergic rhinitis assessed by symptoms, rhinomanometry and nasal cytology. F Allergy Clin Immunol 1990;86: 221-30.

9 Dolovich J, Anderson M, Chodirker W, et al. Fluticasone propionate: a large multicenter trial. Respir Med 1990; 84(Suppl A):31-2.

10 Dolovich J, O'Connor M, Stepner N, et al. Double-blind comparison of intranasal fluticasone propionate $200 \mu \mathrm{g}$ once daily with $200 \mu \mathrm{g}$ twice daily in the treatment of patients with severe seasonal allergic rhinitis to ragweed. patients with severe seasonal

11 Harding SM. The human pharmacology of fluticasone propionate. Respir Med 1990;84(Suppl A):25-9.

12 Watson WTA, Becker AB, Estelle F, et al Treatment of allergic rhinitis with intranasal corticosteroids in patients with mild asthma: effect of lower airway responsiveness. $\mathcal{F}$ Allergy Clin Immunol 1993;91:97-101.
13 American Thoracic Society. Standards for the diagnosis and care of patients with chronic obstructive pulmonary disease Birnie D, Schwartzenberg GWS, Hop WCJ, et al. Does the outcome of the tidal breathing and dosimeter methods of assessing bronchial responsiveness in children depend on age? Thorax 1990;45:199-202.

15 Verberne AAPH, Hop WCJ, Bos AB, et al. Effects of a single dose of inhaled salmeterol on baseline airway caliber and metacholine induced airway obstruction in asthmatic children. F Allergy Clin Immunol 1993;91:127-34.

16 Dodds LJ. Effects of information leaflets on compliance with antibiotic therapy. Pharm F 1986;236:48-57.

17 Barbato A, Pisetta F, Ragusa A, et al. Modification of bronchial hyperreactivity during pollen season in children allergic to grass. Ann Allergy 1987;58:121-4.

18 Boulet LP, Cartier A, Thompson NC, et al. Asthma and increases in non-allergic bronchial responsiveness from seasonal pollen exposure. f Allergy Clin Immunol 1983;71: 399-406.

19 Sotomayor H, Badier M, Vervloet D, et al. Seasonal increase of carbachol airway responsiveness in patients allergic to of carbachol airway responsiveness in patients

grass pollen. Am Rev Respir Dis 1984;130:56-63.
20 Rosenthal RR, Bleecker ER, Norman PS, et al. Effect of environmental antigen on cholinergic hyperreactivity. Chest 979;75(Suppl):228-9.

21 Barbato A, Pisetta F, Nobiato M. Influence of aeroallergens on bronchial reactivity in children sensitized to grass pollens. Ann Allergy 1986;56:138-41.

22 Graff-Lonnevig V, Hedgkin G. The effect of ketotifen on bronchial hyperreactivity in childhood asthma. F Allergy Clin Immunol 1985;76:59-63.

23 Iwata $M$, Tano $M$, Sugiyama S, et al. Effect of azelastine, an antiasthmatic drug, on bronchial hyperresponsiveness in patients with bronchial asthma. Chest 1989;95:1231-4.

24 Van Ganse E, Kaufman L, Derde MP, et al. Effects of antihistamines in adult asthma: a meta-analysis of clinical histamines in adult asthma: a me

25 Corren J, Harris A, Fourre J, et al. Efficacy and safety of Claritin-D in patients with seasonal allergic rhinitis (SAR) and asthma. Ann Allergy Asthma Immunol 2000 (in press).

26 Grant JA, Nicodemus CF, Findlay SR, et al. Cetirizine in patients with seasonal allergic rhinitis and concominant asthma; prospective, randomized, placebo-controlled trial. f Allergy Clin Immunol 1995;95:923-32.

27 Camelleri AE. Information leaflets in the rhinitis clinic? $f$ Laryngol Otol 1991;105:282-4. 